\title{
Influence of the Gas Velocity on the Temperature Homogeneity of Transducers for Gas Sensors
}

\author{
Julia Herrmann, Thomas Kern, Gunter Hagen, Ralf Moos \\ Department of Functional Materials, University of Bayreuth, D-95440 Bayreuth, Germany
}

\begin{abstract}
High-temperature gas sensors need homogeneous heating in the functional sensing area. Varying gas flow characteristics lead to cross-effects and signal instability. Utilizing a special sensor transducer, these effects can be proven experimentally. FEM modelling further supports the understanding. Proper protection caps solve cross-sensitivity problems but also cause decreasing sensor response.
\end{abstract}

Keywords: Exhaust gas sensors, Thermoelectric gas sensors, FE simulation.

\section{Introduction}

Gas sensors provide necessary information in several processes of daily life. Air quality measurements for safety reasons, exhaust gas detection for controlling aftertreatment systems or for on-board-diagnosis, evaluation of flue gas in biomass combustion processes for modern energy technology but also medical breath analysis require stable and reliable sensor signals. Most chemical sensors therefore must be operated at defined temperatures.

In fact, this temperature has to be homogeneous over the whole sensor area (i.e. that region of the sensing element, where the gas interacts with the applied gas sensitive functional materials) and may not be influenced by external parameters like, e.g., gas velocity. The temperature and its homogeneity must be kept constant also during dynamic changes of the environmental conditions, i.e. in all possible working points.

On the other hand, gas contact is highly desired for both fast response and high sensitivity. In the present contribution, general aspects concerning sensor housing and heating are highlighted. Basically, for experimental investigations, a thermoelectric sensor device is used which is an ideal candidate to identify typical problems. As well, simulations were made to verify the results.

\section{Setup: Sensor and Experiments}

The here used sensor transducers are derived from thermoelectric hydrocarbon sensors, developed for automotive exhaust measurements [1] and investigated also in the flue gas of wood burning processes [2]. The sensor measures a temperature difference between two areas within the sensor tip and therefore gives a direct measure of the temperature homogeneity in the interesting region (fig. 1).

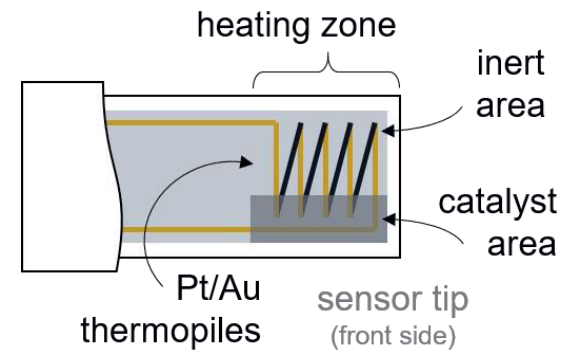

Figure 1: Sketch of the sensor tip.

If one of these areas is catalytically activated and the other region is covered by an inert layer, exothermic reactions generate a temperature gradient between both areas. This gradient is measured by serially connected screen-printed thermopiles in form of a thermovoltage in the $\mu \mathrm{V}$-range. Without test gas, temperature gradients (coming from changing flow conditions any kind of inhomogeneous heating or thermal flow) are measured as well. In former work, it was shown that laminar and symmetric flow characteristics around the sensor tip might avoid cross sensitivities [3]. Here, the described thermoelectric sensors are operated at $600{ }^{\circ} \mathrm{C}$. This absolute sensor temperature is adjusted with a thick-film heater, connected in four-wire technique and located on the reverse side of the substrate. The four-wire resistance is kept constant and so is also the temperature on the reverse side. To evaluate the gas flow 
influence on the front (sensing) side of the substrate, measurements were conducted with different flow rates (compressed air, gas flow directly facing the sensors front side) and under variation of the mounting position (rotating the sensor by a defined angle concerning the gas flow direction) as well as the use of different housings.

Finite-Element-Simulations were made with COMSOLMultiphysics. The here presented data show the gas velocity distribution, regarding the effects around and inside a porous (sintered metal) protection cap (fig. 2). Test gas measurements with propene $\left(\mathrm{C}_{3} \mathrm{H}_{6}\right)$ show the influence of this protection cap concerning the gas flow but also sensitivity.

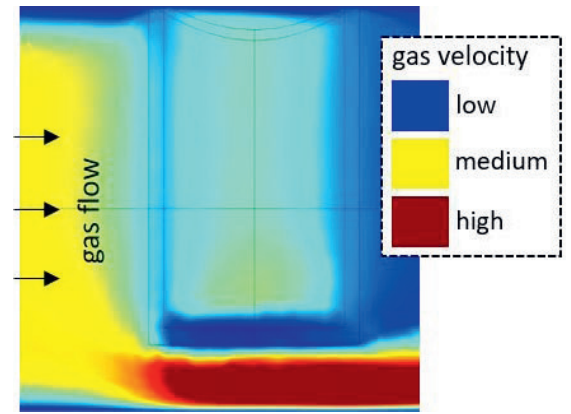

Figure 2: FEM-modelling of the gas flow characteristic around the housed sensor tip.

\section{Results and Conclusions}

In a first experiment, sensors were operated in different gas flows without a protection cap. The gas flow varied between 0 and $50 \mathrm{l} / \mathrm{min}$ in a tube of 1" diameter. Sensors were mounted from above ("hanging") with its front side facing the gas flow. Even here, within the sensor tip temperature differences of $5{ }^{\circ} \mathrm{C}$ occure. It gets worse by rotating the sensor orientation $+/-45^{\circ}$ (gradients up to $20^{\circ} \mathrm{C}$ ), coming from nonsymmetric cooling effects. By using a porous protection cap around the sensor, this influence is minimized $\left(<0.5{ }^{\circ} \mathrm{C}\right)$ due to low and more homogeneous gas velocities inside the cap (fig. 2). Secondly, the sensor results were measured with admixing test gas (mounting in $0^{\circ}$-position, i.e. directly facing the gas flow, but under 20 and $40 \mathrm{l} / \mathrm{min}$ ). Results were evaluated regarding the sensor sensitivity $S$, with is the slope in the characteristic curve (fig. 3). It could be shown that without the protection cap, both parameters - the gas flow as well as the test gas concentration influence the sensor signal in similar height. Using the cap, the gas flow influence can be avoided, but a loss in sensitivity must be taken into account. Reasons therefore could be heterogeneous catalytic effects on the cap surface or transport controlled diffusion through the porous cap. Several other types of protection caps were also tested and simulated. It is necessary to find an ideal configuration for sensor mounting, heater design and protection cap depending on the particular application.

These findings should be transferred to all other kinds of chemical gas sensors where flow characteristics and heating play a role. Temperature gradients on the sensors surface influence directly all mechanistic processes in resistive, amperometric, mixed-potential or potentiometric type sensors.

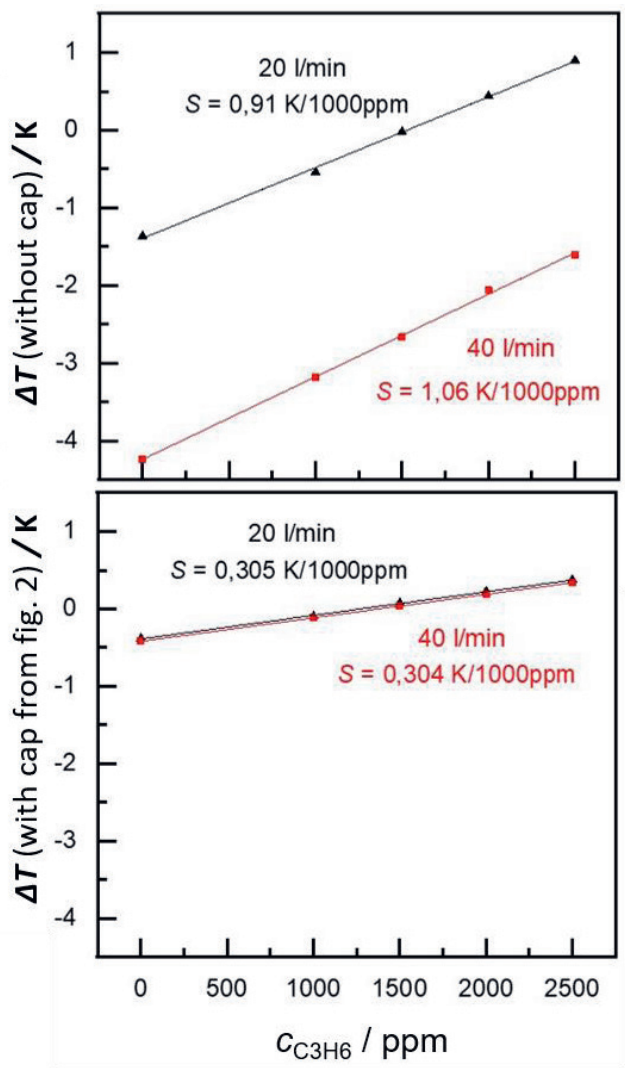

Figure 3: Sensor characteristic with and without cap depending on the gas flow.

\section{References}

[1] S. Wiegärtner, G. Hagen, J. Kita, W. Reitmeier, M. Hien, P. Grass, R. Moos, Thermoelectric hydrocarbon sensor in thick-film technology for on-board-diagnostics of a diesel oxidation catalyst, Sens. Act. B, 214 (2015) 234-240. doi: 10.1016/j.snb.2015.02.083.

[2] B. Ojha, G. Hagen, H. Kohler, R. Moos, Exhaust Gas Analysis of Firewood Combustion Processes: Application of a Robust Thermoelectric Gas Sensor, Proceedings 1 (2017) 457. doi: 10.3390/proceedings 1040457.

[3] G. Hagen, A. Harsch, R. Moos, A pathway to eliminate the gas flow dependency of a hydrocarbon sensor for automotive exhaust applications, J. Sens. Sens. Syst. 7 (2018) 7984. doi: 10.5194/jsss-7-79-2018. 